\section{A2.18 THE EXPRESSION OF THE TRANSCRIPTION FACTOR PPAR-GAMMA IS SIGNIFICANTLY REDUCED IN THE SALIVARY GLAND EPITHELIAL CELLS FROM PATIENTS WITH PRIMARY SJÖGREN'S SYNDROME}

doi:10.1136/annrheumdis-2013-203215.18

Aigli G Vakrakou, Vicky Gourzi, Efstathia Kapsogeorgou, Menelaos N Manoussakis. Dpt. Pathophysiology, University of Athens, Greece

Background and Objectives PPAR-gamma is an essential transcription factor that apart from participating in the regulation of genes associated with lipogenesis, it exerts significant anti-inflammatory actions. PPAR-gamma has been implicated in the pathogenesis of human autoimmune diseases that are characterised by the inflammatory damage of epithelial cells. More specifically, dramatically reduced expression of PPAR-gamma has been shown in the epithelial cells of the intestinal tract and of the biliary tree of patients with ulcerative colitis and primary biliary cirrhosis, respectively. Primary Sjögren's syndrome (SS or autoimmune epithelitis) is characterised by chronic inflammatory lesions mainly affecting epithelial tissues and is associated with systemic autoimmune responses and the chronic intrinsic activation of salivary gland epithelial cells (SGEC). Thus, SGEC are probably both the target and the inducer of inflammatory responses. In this context, we aimed to investigate the levels of constitutive expression of PPAR-gamma in cultured non-neoplastic SGEC lines from SS patients and non-SS controls, as well as the patterns of PPAR-gamma expression following cellular activation.

Materials and Methods To examine the levels of mRNA expression of PPAR-g, total RNA was isolated from long-term cultured non-neoplastic SGEC and from peripheral blood mononuclear cells (PBMCs) of 18 SS patients and 11 non-SS disease controls. The expression of PPAR-gamma was studied by Real-time PCR with primers specific for PPAR-gamma and the reporter gene HPRT1 and analysed by the ddCt method. To evaluate the effect of epithelial activation in the expression of PPAR-g, SGEC from non-SS controls were stimulated with specific ligands of TLR-3 (Polyinosinic-polycytidylic acid, PolyI:C, $5 \mathrm{mg} / \mathrm{ml}$ ), TLR-4 (lipopolysaccharide, LPS, $1 \mathrm{mg} / \mathrm{ml}$ ) receptor and with the cytokine IFN-gamma (500 U/ml). Results PPAR-gamma mRNA expression was significantly reduced in the SGEC from patients with SS, compared to controls $(p=0.0001)$. In contrast, no difference was found in PPAR-gamma expression in PBMCs between patients and controls. The activation of cultured SGEC by stimulation with PolyI:C, LPS and IFN-g resulted in a significant down-regulation of PPAR-gamma mRNA expression (in all cases; by $\approx 80 \%$ at 12 hours, $p=0.0001$ ).

Conclusions Our findings indicate that the expression of PPARgamma in human epithelial cells is significantly reduced following activation via TLRs and the Th1 cytokine INF-gamma. Furthermore, the present study demonstrates for the first time the significantly reduced expression of PPAR-gamma in the SGEC of SS patients. This finding likely owes to the chronic intrinsic activation, which characterises the epithelia of SS patients.

\section{A2.19 THE ROLE OF microRNA 155 IN INNATE IMMUNITY AND ARTHRITIS}

doi:10.1136/annrheumdis-2013-203215.19

Victoria Saferding, Eliana Goncalves-Alves, Antonia Puchner, Josef Smolen, Kurt Redlich, Stephan Blüml. Medical University Vienna, Dpt. of Rheumatology

Background MicroRNA 155 (miR155) has been demonstrated to be essential for the development of collagen induced arthritis by controlling the generation of autoreactive $\mathrm{T}$ and $\mathrm{B}$ cells. However, the contribution of miR155 in innate immune cells is not known.

Materials and Methods We analysed activation and cytokine production of macrophages and dendritic cells (DCs) in vitro and in vivo. We analysed T-cells stimulatory capacity of DCs. We crossed miR155 deficient mice into hTNFtg mice and analysed arthritis development clinically as well as histologically.

Results MiR155 deficiency did not alter the expression of costimulatory molecules or MHCII expression after stimulation of macrophages and DCs in vitro and in vivo. We also Facs-sorted DCs after stimulation with LPS in vivo and determined the production of proinflammatory cytokines such as IL-23, IL-6 as well as TNF. We did not detect differences between wt and miR155\% mice. In addition, the T cell stimulatory capacity of wt and $\operatorname{miR} 155^{-/}$was identical. When we analysed hTNFtg/miR155\% mice compared to wt mice, we did not detect differences in the clinical signs and symptoms of arthritis. Histologically, we even found slightly increased synovial inflammation in hTNFtg/miR155/- mice compared to wt mice.

Conclusions In contrast to the pivotal role of miR155 in autoimmunity requiring the adaptive immune system, the role of miR155 in innate immunity seems to be limited. This is emphasised by the fact that miR155 hardly influences the course of TNF-driven arthritis, which is mainly dependent on components of the innate immune system.

\section{A2.20 THE ROLE OF SERUM FACTORS AND TOLL-LIKE RECEPTOR SIGNALING IN THE INDUCTION OF PROFIBROTIC TIMP-1 BY MONOCYTES IN SYSTEMIC SCLEROSIS}

doi:10.1136/annrheumdis-2013-203215.20

${ }^{1}$ Marzena Ciechomska, ${ }^{1}$ Christiaan A Huigens, ${ }^{2}$ Thomas Hügle, ${ }^{3}$ Bridget Griffiths, ${ }^{4}$ Timothy RDJ Radstake, 'Sophie Hambleton, 'Steven O'Reilly, 'Jacob M van Laar. 'Musculoskeletal Research Group, Institute of Cellular Medicine, Newcastle University, Newcastle Upon Tyne, UK; '2Dept. of Rheumatology, University Hospital Basel, Switzerland; ${ }^{3}$ Dept. of Rheumatology, Freeman Hospital, Newcastle Upon Tyne, UK; ${ }^{4}$ Dept. of Rheumatology and Clinical Immunology, University Medical Centre Utrecht, The Netherlands

Background and Objectives Systemic sclerosis (SSc) is an autoimmune disease characterised by fibrosis, vascular dysfunction and abnormal activation of immune cells including monocytes. Monocytes along with fibroblasts play an important role in the production of profibrotic factors such as IL-6 and TIMP-1 (tissue-inhibitor of metalloproteinase-1). TIMPs are specific inhibitors of matrix metalloproteinases (MMPs) regulating extracellular matrix (ECM) turnover. Importantly, the balance between TIMPs and MMPs is altered in most pathological stages including SSc and is associated with abnormal ECM formation. However, the exact factors which drive both profibrotic TIMP-1 secretion are not fully defined.

We aim to test whether circulating monocytes from SSc patients produce TIMP-1 in response to TLR activation and serum factors, which contributes to excessive matrix deposition and consequently disease progression.

Materials and Methods 25 patients with SSc, one IRAK4 deficient patient and $20 \mathrm{HC}$ (healthy control) were included in this study. Peripheral blood monocytes were further separated by CD14+ microbeads. Production of TIMP-1, IL- 6 by monocytes was determined by ELISA, qRT-PCR or functional assay in response to either panel of conventional TLR agonists or HC and SSc sera. Skin section from SSc patient was stained with CD14+ and TIMP-1 antibodies and further analysed by confocal microscopy.

Results TIMP-1 production by monocytes was observed in the SSc skin section and was upregulated in SSc patients compared to HC. Incubation of $\mathrm{HC}$ monocytes with SSc sera resulted in functionally active TIMP-1 production. However, pre-treatment with MyD88 inhibitor, but not control peptide, decreased TIMP-1 secretion. Furthermore, SSc-mediated TIMP-1 induction by monocytes was attenuated when Fc $\gamma R$ was blocked and also when SSc sera were treated with DNA/RNA endonuclease prior to stimulation. This indicates that SSc sera contain RNA/DNA agonists inducing TIMP-1 production. Indeed, direct treatment of $\mathrm{HC}$ and SSc 
monocytes with a panel of TLR ligands demonstrated strong TIMP-1 and IL-6 production following triggering with TLR8 agonists (ssRNA). TLR8-mediated TIMP-1 production was reduced in monocytes from a patient with a genetic TLR signalling defect or $\mathrm{HC}$ monocytes cultured with MyD88 inhibitory peptide. Furthermore, matrix assay of TLR8 stimulated monocytes also confirmed functional TIMP-1 secretion, as matrix metalloproteinase-1 activity was significantly inhibited.

Conclusions This study indicates a potential link between SSc serum factors and TLR signalling resulting in excessive TIMP-1 secretion by circulating monocytes from SSc patients.

\section{A2.21 TOLL-LIKE RECEPTOR DEPENDENT AUTOANTIGENS AND VESICLES FROM P. GINGIVALIS IN ANIMAL MODELS OF RA TO MODULATE COLLAGEN AND COLLAGEN ANTIBODY INDUCED ARTHRITIS}

doi:10.1136/annrheumdis-2013-203215.21

${ }^{2 B}$ Marklein, ${ }^{2} \mathrm{M}$ Sohn, ${ }^{1 Z}$ Konthur, ${ }^{2} \mathrm{~K}$ Grimm, ${ }^{3} \mathrm{G}$ Steiner, ${ }^{4} \mathrm{~F}$ Apparailly, ${ }^{2} \mathrm{G}-\mathrm{R}$ Burmester, ${ }^{1.2} \mathrm{~K}$ Skriner. 'Max-Planck-Institute for Molecular Genetics, Berlin, Germany; ${ }^{2}$ Charité University Medicine, Department of Rheumatology and Clinical Immunology, Humboldt University and Free University, Berlin, Germany; ${ }^{3}$ University Departments of the Vienna Biocenter. Department of Medical Biochemistry, Dr. Bohrgasse 9/4, A-1030 Vienna, Austria; " IINSERM, U844, Hôpital Saint Eloi, and Université Montpellier I, Montpellier, France

Background and Objectives A variety of animal models suggest that TLR signalling is important in the pathogenesis of RA and the generation of specific autoantibodies. This study was conducted with sera from patients with rheumatoid arthritis, as well as with arthritis animal models to identify identical autoantigens dependent on TLR7 and 9 in human and animal models for disease modifying use. Moreover TLR2 and TLR4 modulating bacterial vesicles from P. gingivalis containing PAD (Peptidyl-Arginine Deiminase) which is involved in citrullination was used to study the TLR2/4 in arthritis.

Materials and Methods Using protein philtre technology (28000 human protein philtre) the autoantigen profile of RA patients, mouse collagen and zymosan induced arthritis, as well as collagen and pristan induced arthritis in rats and TLR7, TLR9 deficient double-deficient and MyoD88 and Tir8 deficient mice of the MRLlpr/lpr background were obtained. Cationic liposomes transferring siRNAs, bacterial vesicles, lipomannan and LPS were used for the validation of their potential as therapeutic target in collagen or collagen antibody induced arthritis (CAIA).

Results We found 18 identical proteins targeted in human and animal situations of arthritis. These data identify mRNA binding hnRNPs proteins which are part of $\mathrm{P}$ bodies, stress granules and components of messenger RNA stability complex as well as CRP binding proteins as target molecules in mice, rats and humans with RA. Moreover, we found MyoD88 independent autoantigens which are not expressed in the thymus or proteins such as high mobility group box proteins 1 and 2 which are MyoD88 independent sensors of nucleic-acid-mediated innate immune responses. Systemic administration of siRNAs with cationic liposomes inhibiting expression of Toll dependent autoantigens overexpressed and targeted by autoantibodies in the human and mouse synovial tissue were used for the validation of their potential to inhibit collagen induced arthritis in $\mathrm{C} 57 \mathrm{BL} / 6 \mathrm{~J}$ mice. Moreover P. gingivalis vesicles containing the PAD induce a mild inflammatory response in the CAIA model of arthritis. $\mathrm{P}$. gingivalis LPS and lipomannan treated animals show a 80\% reduction of athrithis score compared to E coli LPS in a C57BL/6J CAIA model.

Conclusions Systemic blocking of common RNA or DNA binding proteins overexpressed in synovial target tissue appears to modify arthritis. $P$. gingivalis vesicles evolved the ability to intercept and undermine a subset of TLR2/4 signalling events for corrupting innate immunity and modulate RA.

\section{A2.22 TYROSINE PHOSPHORYLATION PATHWAYS IN MYELOID CELL-MEDIATED INFLAMMATORY DISEASES}

doi:10.1136/annrheumdis-2013-203215.22

M Kovács, T Németh, K Futosi, Z Jakus, C Sitaru A, Mócsai. Department of Physiology, Semmelweis University School of Medicine, Budapest, Hungary; Department of Dermatology, University of Freiburg, Germany

Background Tyrosine kinases are major therapeutic targets in cancer but their role in immune-mediated disease pathogenesis is less understood.

Methods Here we tested the role of tyrosine kinases and tyrosine kinase substrates in two autoantibody-mediated disease models, the $\mathrm{K} / \mathrm{BxN}$ serum-transfer arthritis and anti-collagen VII autoantibody-induced blistering skin diseases.

Results Mice genetically deficient of the Syk tyrosine kinase in the hematopoietic compartment were completely protected from autoantibody-induced arthritis and blistering skin disease. Mice lacking three myeloid-specific tyrosine kinases (Hck, Fgr and Lyn) or the tyrosine kinase substrate PLC $\gamma 2$ were also protected from autoantibody-induced diseases. In vitro, Src-family kinases were required for Syk activation by immune complexes and both were further required for activation of PLC $\gamma 2$. The Src-family-Syk-PLC $\gamma 2$ pathway mediated cytokine production by myeloid cells but not neutrophil or monocyte migration per se. Lineage-specific analyses revealed that during autoantibody-induced arhritis, this signalling pathway was required in myeloid cells (particular neutrophils) but not in mast cells or platelets. Finally, Src-family kinases were also required for activation of myeloid cells by monosodium urite crystals and their deficiency attenuated monosdium urate crystal-induced arthritis, indicating that the role of this signalling pathway is not restricted to autoantibody-mediated disease processes.

Conclusions Taken together, the Src-family-Syk-PLC $\gamma 2$ pathway is an important component of both autoantibody-mediated and autoantibody-independent inflammatory disease processes.

\section{A2.23 IMPAIRED NATURAL KILLER CELL FUNCTION IN DOCK8 DEFICIENCY}

doi:10.1136/annrheumdis-2013-203215.23

1.2MC Mizesko, 1.2PP Banerjee, ' ${ }^{2} \mathrm{~L}$ Monaco-Shawver, 1,2EM Mace, ${ }^{3} \mathrm{~W}$ Bernal, ${ }^{4} \mathrm{~J}$ Sawalle-Belohradsky, ${ }^{4 B}$ Belohradsky, ${ }^{4} \mathrm{~V}$ Heinz, ${ }^{5} \mathrm{AF}$ Freeman, ${ }^{3} \mathrm{KE}$ Sullivan, ${ }^{5} \mathrm{SM}$ Holland, ${ }^{6}$ TR Torgerson, ${ }^{7} \mathrm{~W}$ Al-Herz, ${ }^{8} \mathrm{~J}$ Chou, ${ }^{1,2} \mathrm{C} \mathrm{C}$ Hanson, ${ }^{4} \mathrm{MH}$ Albert, ${ }^{8} \mathrm{RS}$ Geha, ${ }^{4} \mathrm{ED}$ Renner, ${ }^{1,2} \mathrm{JS}$ Orange. 'Baylor College of Medicine; ${ }^{2} T e x a s$ Children's Hospital, Houston, TX: ${ }^{3}$ Children's Hospital of Philadelphia Research Institute, Philadelphia, PA, USA; 'University Children's Hospital, Ludwig Maximilian University, Munich, Germany; ${ }^{5}$ National Institute of Allergy and Infectious Diseases, National Institutes of Health Bethesda, MD, USA; 'University of Washington and Seattle Children's Hospital, Seattle, WA, USA; 'Kuwait University and Allergy and Clinical Immunology Unit, Kuwait City, Kuwait, ${ }^{8}$ Boston Children's Hospital, Boston, MA, USA

Introduction DOCK8 mutations are responsible for a rare autosomal recessive immunodeficiency syndrome associated with severe cutaneous viral infections, elevated IgE levels, environmental allergies, autoimmunity, and malignancy. DOCK8 activates CDC42, which is important for cell signalling and actin reorganisation. Natural killer cells play a vital role in tumour surveillance and defence against virally infected cells. NK cell function relies on the accumulation of actin at the NK cell immunologic synapse formed with target cells. Although abnormalities in $\mathrm{T}$ and $\mathrm{B}$ cell function have been described in DOCK8-deficient patients, the role of NK cells in this disease is poorly understood. 